Pacific Journal of Mathematic 


\section{COHESIVE SETS AND RECURSIVELY ENUMERABLE DEDEKIND CUTS}

\section{ROBERT I. SOARE}

In this paper the methods of recursive function theory are applied to certain classes of real numbers as determined by their Dedekind cuts or by their binary expansions. Instead of considering recursive real numbers as in constructive analysis, we examine real numbers whose lower Dedekind cut is a recursively enumerable (r.e.) set of rationals, since the r.e. sets constitute the most elementary nontrivial class which includes nonrecursive sets. The principal result is that the sets $A$ of natural numbers which "determine" such real numbers $\alpha$ (in the sense that the characteristic function of $A$ corresponds to the binary expansion of $\alpha$ ) may be very far from being r.e., and may even be cohesive. This contrasts to the case of recursive real numbers, where $A$ is recursive if and only if the corresponding lower Dedekind cut is recursive.

With each subset $A$ of the set of natural numbers $N$, there is naturally associated a real number in the interval [0,2], namely $\Phi(A)=\sum_{n \in A} 2^{-n}$, and $\Phi(\varnothing)=0$. Fix a one-one effective map from $N$ onto $Q$, the set of rationals in the interval [0,2], and denote the image under this map of an element $n$ by the bold face $n$. Identifying each natural number $n$ with its rational image $\mathbf{n}$, the (lower) Dedekind cut associated with $A$ is simply

$$
L(A)=\{n \mid \mathbf{n} \leqq \Phi(A)\} .
$$

It is well known in recursive analysis [4] that $A$ is recursive if and only if $L(A)$ is recursive, and in this case $\Phi(A)$ is said to be a recursive real number.

From the point of view of recursion theory, however, it is more natural to consider certain wider classes of Dedekind cuts, especially those which are recursively enumerable (r.e.). The most interesting results in recursion theory concern these sets. In going from recursive to recursively enumerable Dedekind cuts, we find that: $A$ r.e. implies $L(A)$ r.e.; but not conversely. (C.G. Jockusch has observed the following simple counter-example to the converse. If $A$ is any r.e. set and if $B=A$ join $\bar{A}=\{2 n \mid n \in A\} \cup\{2 n+1 \mid n \in \bar{A}\}$, then $L(B)$ is r.e., but $B$ is not r.e. unless $A$ is recursive.) It is now natural to ask just how "sparse" the set $A$ can be so that $L(A)$ remains r.e. At the end of $\S 3$ in [8] we indicated how to construct a hyperimmune set $H$ such that $L(H)$ is r.e. We now consider two notions (dominant and hyper- 
hyperimmune) which are natural extensions (as explained in $\$ 2$ ) of the two equivalent properties used to define a hyperimmune set. We will prove that:

(1) There is a set $A$ such that: (i) $A$ is dominant (i.e. the principal function of $A$ dominates every recursive function); (ii) $L(A)$ is r.e.; and (iii) $A$ contains an infinite retraceable subset, and is not hyperhyperimmune.

(2) There is a cohesive (and hence hyperhyperimmune) set $C$ such that $L(C)$ is r.e.

In addition to illustrating the wide range of sets $A$ which can yield r.e. Dedekind cuts, $L(A)$, these results suggest another method of classifying r.e. Dedekind cuts. Recursively enumerable Dedekind cuts appear to defy classification by the usual division of the r.e. sets into such categories as creative or simple, because the dense linear ordering imposed by the rationals prevents any Dedekind cut from being simple or creative (see [8]). We have suggested in [8] a partial classification of r.e. Dedekind cuts using certain classes of fixed point free recursive maps which preserve them. The construction of the dominant set now suggests the notion of an r.e. Dedekind cut being stably recursively enumerable, a requirement which is strictly intermediate between requiring that $A$ be r.e., and requiring merely that $L(A)$ be r.e.

Background material may be found in the references listed at the end of the paper, especially [6] and [7]. We used the standard enumeration of the r.e. sets, $W_{0}, W_{1}, \cdots$, that is obtained by setting $W_{e}=\left\{x \mid(\exists y) T_{1}(e, x, y)\right\}$ for each $e$; and we set $W_{e}^{z}=\left\{x \mid(\exists y)_{<z} T_{1}(e, x, y)\right\}$ for each $e$ and $z$. For natural numbers $x<y, I[x, y]$ will denote the finite set $\{x, x+1, x+2, \cdots, y\}$. We will also used the standard effective indexing of the finite sets, $\left\{D_{x}\right\}$. Namely, if $x_{1}, x_{2}, \cdots, x_{n}$ are distinct natural numbers, and $x=2^{x_{1}}+2^{x_{2}}+\cdots+2^{x_{n}}$, then $D_{x}$ denotes $\left\{x_{1}, x_{2}, \cdots, x_{n}\right\}$, and $D_{0}$ denotes the empty set, $\varnothing$. We use the standard pairing function, $j(x, y)=x+(1 / 2)(x+y)(x+y+1)$, and following Rogers [6] we will let $\langle x, y\rangle$ denote the image $j(x, y)$. If $P(x)$ is a predicate, then $\sim P(x)$ denotes the negation of $P(x)$, and $! x P(x)$ denotes "the unique $x$ such that $P(x)$ holds". For any set $A \subseteq N, \bar{A}$ denotes $N-A$, card $A$ denotes "cardinality of $A$ ", and $\Phi(A)$ denotes the real number $\sum_{n \in A} 2^{-n}$, while $\Phi(\varnothing)=0$. Finally, we write $A \subset{ }^{*} B$ if $B-A$ is finite.

1. Stably recursively enumerable Dedekind cuts. Before defining the notion of a stably r.e. Dedekind cut, it will be convenient to have the following characterization of a r.e. Dedekind cut. (From now on "cut" will always mean Dedekind cut.) A sequence of finite sets, $\left\{A^{s}\right\}$, is said to be canonically r.e. if there is a recursive function 
$f$ such that $A^{s}=D_{f(s)}$ for all $s$.

LEMmA 1.1. For any set $A$, the cut $L(A)$ is r.e. if and only if there is a canonically r.e. sequence of finite sets, $\left\{A^{s}\right\}$, such that

$$
(s)\left[\Phi\left(A^{s+1}\right) \geqq \Phi\left(A^{s}\right)\right], \text { and }
$$

$$
\left.A=\lim _{s} A^{s} \text { (i.e. }(n)(\exists s)(m)_{\leqq n}(t)_{\geqq s}\left[m \in A \Leftrightarrow m \in A^{t}\right]\right)
$$

Proof. If $A$ is recursive the lemma is clear, so we may assume that $A$ is nonrecursive and thus $\Phi(A)$ is nonrational. Now assume that $\left\{A^{s}\right\}$ is canonically r.e. and satisfies (1.1) and (1.2). For each $s$, define the rational $\mathbf{x}_{s}=\Phi\left(A^{s}\right)$. Then $\lim _{s} \mathbf{x}_{s}=\Phi(A)$, and $L(A)$ is r.e. because $U\left\{x_{s}\right\}$ is r.e., and because $y \in L(A) \Leftrightarrow(\exists s)\left[\mathbf{y} \leqq \mathbf{x}_{s}\right]$, since $\Phi(A)$ is nonrational.

Conversely, assume $L(A)$ is r.e., say $L(A)=W_{e}$. For every $s$ such that $W_{e}^{s} \neq \varnothing$, define $\mathbf{x}_{s}=\max \left\{\mathbf{y} \mid y \in W_{e}^{s}\right\}$, and let $B^{s}$ be the recursive set such that $\Phi\left(B^{s}\right)=\mathbf{x}_{s}$. Let $A^{s}=B^{s} \cap I[0, s]$. Note that $B^{s}$ is recursive since $\mathbf{x}_{s}$ is rational, and $B^{s}$ is unique if whenever a rational has two distinct binary expansions, we always favor the expansion ...1000... instead of ...0111 ... (Since for each $x, \mathbf{x}$ is effectively presented as a quotient of natural numbers, we can effectively recognize this case.) Clearly, the sequence $\left\{A^{s}\right\}$ satisfies (1.1) and (1.2).

In general there is no further restriction upon these sets $A^{s}$, so that in particular an element $n$ may appear and disappear in subsequent sets many times (at most $2^{n+1}$ ) as long as

$$
(s)\left[n \in A^{s}-A^{s+1} \Rightarrow(\exists y)\left[y \in A^{s+1}-A^{s} \& y<n\right]\right.
$$

so that $\Phi\left(A^{s+1}\right) \geqq \Phi\left(A^{s}\right)$ holds.

In view of this we define an r.e. cut $L(A)$ to be stably recursively enumerably (s.r.e.) if there is a canonically r.e. sequence of finite sets $\left\{A^{s}\right\}$ satisfying (1.1) and (1.2) as well as

$$
(n)(s)(t)_{>s}\left[n \in A^{s}-A^{s+1} \Longrightarrow n \notin A^{t}\right] .
$$

If the set $A$ itself is r.e., say $A=W_{e}$, then $L(A)$ is clearly s.r.e. because we may take $A^{s}=W_{e}^{s}$ so that the antecedent in (1.3) never holds. The converse, however, is false by Jockusch's example $L(B)$ given earlier which is easily seen to be s.r.e. but $B$ is not necessarily r.e.

Furthermore, Theorems 1.2 and 3.1 together will imply that not every r.e. cut is s.r.e., and hence that the requirement that $L(A)$ be stably r.e. is strictly intermediate between requiring that $A$ be r.e., and requiring merely that $L(A)$ be r.e. Theorem 1.2 proves that if $A$ is infinite and $L(A)$ is s.r.e. then $A$ contains an infinite retraceable subset. Theorem 3.1 proves that there is a cohesive set $C$ such that 
$L(C)$ is r.e. Since no cohesive set contains an infinite retraceable subset (Rogers [6], Exercise 12-48), $L(C)$ cannot be s.r.e.)

Dekker and Myhill [1] define a set $R$ to be retraceable if there is a partial recursive function $f$ such that $f\left(r_{0}\right)=r_{0}$, and $f\left(r_{n+1}\right)=r_{n}$ for all $n$, where $r_{0}, r_{1}, \cdots$, are the elements of $R$ in ascending order. Given such an $f$, for each $x$ in the domain of $f$, we adopt the convention that $f^{0}(x)=x$, and define the set,

$$
\hat{f}(x)=\left\{y \mid(\exists n)\left[f^{n}(x)=y\right]\right\} \text {. }
$$

Theorem 1.2. If $A$ is infinite, and $L(A)$ is stably r.e., then $A$ contains an infinite retraceable subset $B$, which is retraceable by a finite-one, partial recursive fuction $f$.

Proof. Assume that $A$ is infinite and that $\left\{A^{s}\right\}$ is a canonically r.e. sequence of finite sets satisfying (1.1), (1.2) and (1.3). At each stage $s$, the partial recursive retracing fuction $f$ will be defined on at most a finite number of elements. Let $a_{0}=\mu x[x \in A]$, and $s_{0}=$ $\mu s\left[a_{0} \in A^{s}\right]$. Our construction begins at stage $s=s_{0}$.

Stage $s=s_{0}$. Let $a_{1}^{s_{0}}, a_{2}^{s_{0}}, \cdots$, be the elements of $A^{s_{0}}$ which are greater than $a_{0}$, listed in ascending order. Define $f\left(a_{0}\right)=a_{0}$, and $f\left(a_{i+1}\right)=a_{i}$ for all $i$.

Stage $s>s_{0}$. Let $a_{1}^{s}, a_{2}^{s}, \cdots$ be the elements of $A^{s}-\bigcup_{t<s} A^{t}$ listed in ascending order. Define

$$
\begin{aligned}
& f\left(a_{1}^{s}\right)=! x\left[x<a_{1}^{s} \& \hat{f}(x) \subseteq A^{s}\right. \\
& \left.\quad \&(y)\left[y<a_{1}^{s} \& \hat{f}(y) \subseteq A^{s} \Rightarrow \Phi(\hat{f}(x)) \geqq \Phi(\hat{f}(y))\right]\right] \\
& f\left(a_{i+1}^{s}\right)=a_{i}^{s}, \text { for all } i>1 .
\end{aligned}
$$

Clearly $f$ is partial recursive and finite-one because of our conditions on the sequence $\left\{A^{s}\right\}$.

We now exhibit an infinite subset of $A$, namely $B=\left\{b_{0}, b_{1}, \cdots\right\}$, which is retraced by $f$. Define $b_{0}=a_{0}$,

$$
b_{n+1}=\mu x\left[f(x)=b_{n} \& x>a_{0}\right] .
$$

Clearly, $B$ is retraced by $f$, and $B$ is infinite since $A$ is infinite. To show $B \cong A$ we first define $s(m)=\mu s\left[b_{m} \in A^{s} \& s \geqq s_{0}\right]$. We prove simultaneously by induction on $m$ that,

$$
\begin{gathered}
(m)\left[b_{m} \in A\right] \\
(t)_{\geqq s(m)}(n)_{\geqq_{m}}\left[n \in A \Leftrightarrow n \in A^{t}\right] .
\end{gathered}
$$

These are clearly true for $m=0$. Assume true for all $m \leqq p$. Now $s(p+1) \geqq s(p)$ because $f\left(b_{p+1}\right)=b_{p}$. Suppose $n \in A^{t+1}-A^{t}$ for some 
$n<b_{p+1}$ and some $t \geqq s(p+1)$. Let $n^{\prime}$ be the least such $n$, and $t^{\prime}$ the least corresponding $t$. By inductive hypothesis $b_{p}<n^{\prime}$, but by stability of $\left\{A^{s}\right\}, n^{\prime} \notin \bigcup_{u \leqq t^{\prime}} A^{u}$. Thus at stage $t^{\prime}+1$ we must define $f\left(n^{\prime}\right)=b_{p}$, contradicting the definition of $b_{p+1}$. Hence (1.5) holds for $m=p+1$. But then $b_{p+1} \in A$ by (1.5) and (1.1) since $b_{p+1} \in A^{s(p+1)}$.

2. A dominant set with recursively enumerably lower cut: Following Martin [3], we say that a function $f$ dominates a function $g$, if for all but finitely many $n, f(n) \geqq g(n)$. The principal function of an infinite set $A$ is that function which enumerates the members of $A$ in order of magnitude without repetition. A function $f$ dominates an infinite set $A$ if $f$ dominates the principal function of $A$.

We define an infinite set $A$ to be dominant if the principal function of $A$ dominates every recursive function. It is easily seen that $A$ is dominant if and only if the principal function of $A$ dominates every infinite r.e. set, and we will use this property in the proof of Theorem 2.1. (Martin [3] used no name for a dominant set, but called a set $A$ dense if $\bar{A}$ is either finite or dominant.)

A set $H$ is said to be hyperimmune if there is no recursive function $f$ such that for all $x$ and $y$,

$$
D_{f(x)} \cap H \neq \varnothing \&\left[x \neq y \Rightarrow D_{f(x)} \cap D_{f(y)}=\varnothing\right],
$$

or equivalently if no recursive function dominates the principal function of $H$ (Rice [4]). A set $H$ is hyperhyperimmune if there is no recursive function $f$ such that for all $x$ and $y$,

$$
W_{f(x)} \cap H \neq \varnothing \& W_{f(x)} \text { is finite } \&\left[x \neq y \Rightarrow W_{f(x)} \cap W_{f(y)}=\varnothing\right] .
$$

The notions of hyperhyperimmune and dominant represent respectively the strengthenings of the two equivalent conditions of hyperimmunity. Since it is possible [8] to construct a hyperimmune set $H$ such that $L(H)$ is r.e., it is natural to attempt to obtain the same conclusion for these two "sparser" types. We construct below a dominant set $A$ such that $L(A)$ is stably r.e. By Theorem 1.2, $A$ contains an infinite subset $B$ retraced by a finite-one, partial recursive retracing function, and hence $A$ is not hyperhyperimmune (by the same proof as in Rogers [6], Exercise 12-48 (a)). (Martin [2], p. 275 constructs a co-r.e. set $A$ which is dominant but not hyperhyperimmune. Of course, our set $A$ cannot be co-r.e. since $L(A)$ would be recursive.)

For each $s$ and $e$, we define the partial recursive function $h(s, e, n)$ to be that function which enumerates the members of $W_{e}^{s}$ in ascending order and is undefined for $n \geqq$ cardinality of $W_{e}^{s}$ (denoted card $W_{e}^{s}$ ). (Since the first element of $W_{e}^{s}$ is given by $h(s, e, o)$, the function will be defined only for $n<$ card $W_{e}^{s}$.) Now $\lim _{s} h(s, e, n)$ clearly exists for 
each $e$ and $n<$ card $W_{e}^{s}$, and will be denoted by the partial function $h(e, n)$, which is the principal function of $W_{e}$ if $W_{e}$ is infinite. Note also that,

$$
(s)(e)(n)[h(s, e, n) \geqq h(s+1, e, n) \geqq h(e, n)]
$$

whenever the functions are defined.

THEOREM 2.1. There is a dominant set $A$ such that $L(A)$ is stably recursively enumerable.

(Intuitively, one may think of the following proof as an attempt to satisfy an infinite number of "requirements", where requirement $\langle e, i\rangle$, denoted $R_{\langle e, i\rangle}$, states that

$$
(n)[\langle e, i\rangle\langle n \leqq\langle e, i+1\rangle \Rightarrow a(n) \geqq h(e, n)],
$$

where $a(n)$ is the principal function of $A$. We say that requirement $R_{\langle e, i\rangle}$ has higher priority than requirement $R_{\langle x, y\rangle}$ if $\langle e, i\rangle\langle\langle x, y\rangle$. In Lemma 2.4 we will prove that if $W_{e}$ is infinite, then for every $i$, $R_{\langle e, i\rangle}$ is satisfied, and thus $a(n)$ dominates $h(e, n)$. To convert our proof into a "movable markers" argument as in Rogers [6] one need merely imagine that a "marker" $\Lambda_{\langle e, i\rangle}$ is uniquely associated with $R_{\langle\epsilon, i\rangle}$ for each $\langle e, i\rangle$, and that $v(s, e, i)$ denotes the integer occupied by marker $\Lambda_{\langle e, i\rangle}$ at stage s. Then for example, (2.2) states that the markers are always arranged in order according to the priority of $R_{\langle e, i\rangle}$, and the definition of $v(s+1, e, i)$ may be viewed as a description of how the markers move.)

Proof. We will construct by stages a canonically r.e. sequence of finite sets, $\left\{A^{s}\right\}$, which satisfies (1.1), (1.2) and (1.3), and such that if $a(n)$ is the principal function of the set $A=\lim _{s} A^{s}$, then $a(n)$ dominates $\lambda n h(e, n)$ whenever $W_{e}$ is infinite. Simultaneously, we will define by stages a recursive function $v(s, e, i)$ such that for all $s, e, i, x$ and $y$,

$$
\begin{gathered}
v(s, e, i)\langle v(s, x, y) \Leftrightarrow\langle e, i\rangle\langle\langle x, y\rangle \\
v(s, e, i) \leqq v(s+1, e, i)
\end{gathered}
$$

Define $A^{0}=\varnothing$, and $v(0, e, i)=\langle e, i\rangle$ for all $e$ and i.

Stage $s \geqq 0$. We say that the integer $\langle e, i\rangle$ is eligible at stage $s$ if $v(s, e, i) \notin A^{s}$ and card $\left.W_{e}^{s}\right\rangle\langle e, i+1\rangle$. If no integer is eligible at stage $s$ then set $A^{s+1}=A^{s}$ and $v(s+1, e, i)=v(s, e, i)$ for all $e$ and $i$, and go to stage $s+1$. Otherwise, let $\left\langle e_{s}, i_{s}\right\rangle$ denote the least integer eligible at stage $s$, and define, 


$$
A^{s+1}=\left(A^{s} \cap I\left[0, v\left(s, e_{s}, i_{s}\right)\right]\right) \cup\left\{v\left(s, e_{s}, i_{s}\right)\right\} .
$$

Note that in either case,

$$
\Phi\left(A^{s+1}\right) \geqq \Phi\left(A^{s}\right)
$$

because in the second case $\left\langle e_{s}, i_{s}\right\rangle$ eligible at stage $s$ implies that $v\left(s, e_{s}, i_{s}\right) \notin A^{s}$.

In order to insure stability of $A$ as well as (2.2) and (2.3) we define a predicate $V(t+1, e, i, n)$ which specifies certain integers $n$ which are available as values for $v(t+1, e, i)$. (It will be clear that the function $v(t, e, i)$ is recursive by recursion first upon $t$ and then upon $\langle e, i\rangle$ because $v(t+1, e, i)$ is uniformly recursive in $V(t+1, e, i, n)$ which itself is uniformly recursive in $v(t, e, i)$ and $v(t+1, x, y)$ for $\langle x, y\rangle\langle\langle e, i\rangle$.)

$$
\begin{aligned}
V(t+1, e, i, n) \equiv & (u)_{\leqq t}\left[n \notin A^{u} \& n \geqq v(t, e, i)\right. \\
& \&(x)(y)[\langle x, y\rangle<\langle e, i\rangle \Rightarrow v(t+1, x, y)<n]] .
\end{aligned}
$$

We now complete our construction by defining at stage $s$,

$$
v(s+1, e, i)= \begin{cases}v(s, e, i) & \text { if }\langle e, i\rangle \leqq\left\langle e_{s}, i_{s}\right\rangle \\ \mu n\left[n \geqq h\left(s, e_{s},\langle e, i\rangle\right)\right. & \text { if }\left\langle e_{s}, i_{s}\right\rangle\left\langle\langle e, i\rangle \leqq\left\langle e_{s}, i_{s}+1\right\rangle\right. \\ \& V(s+1, e, i, n)] & \\ \mu n V(s+1, e, i, n) & \text { if }\left\langle e_{s}, i_{s}+1\right\rangle\langle\langle e, i\rangle .\end{cases}
$$

Note that the second and third clauses of $V$ guarantee that $v(s, e, i)$ satisfies (2.3) and (2.2) respectively. (Notice how by the second clause in the definition of $v$ we attempt to satisfy requirement $R_{\left\langle e_{s}, i_{s}\right\rangle}$ at stage s.) Furthermore, we have for all $s, e, i$ and $n$,

$$
n \in A^{s+1}-A^{s} \Leftrightarrow n=v\left(s, e_{s}, i_{s}\right)
$$

$$
\begin{gathered}
v(s, e, i)<v(s+1, e, i) \Rightarrow(\exists m)\left[m<v(s, e, i) \& m \in A^{s+1}-A^{s}\right] \\
n \in A^{s+1}-A^{s} \Rightarrow(t)_{>s}\left[n=v(t, e, i) \Rightarrow\langle e, i\rangle=\left\langle e_{s}, i_{s}\right\rangle\right] \\
(t)_{>s}\left[v\left(t, e_{t}, i_{t}\right)<v\left(s, e_{s}, i_{s}\right) \Rightarrow v\left(s, e_{s}, i_{s}\right)<v\left(t+1, e_{s}, i_{s}\right)\right]
\end{gathered}
$$

where (2.8) is considered vacuous unless $\left\langle e_{s}, i_{s}\right\rangle$ and $\left\langle e_{t}, i_{i}\right\rangle$ are defined. Clearly (2.6) follows from the definition of $v(s+1, e, i)$ and in fact $m=v\left(s, e_{s}, i_{s}\right)$ by (2.5). To prove (2.7) fix $s$ and suppose for some $n$ that $n \in A^{s+1}-A^{s}$. Then $n=v\left(s, e_{s}, i_{s}\right)$. But $n \in A^{s+1}$ implies $(t)_{>s} \sim V(t, e, i, n)$. Thus, if $n=v(t, e, i)$ for some $e$ and $i$, and some $t>s$, it can only be through the first clause in the definition of $v(t, e, i)$. It follows by an easy induction on $t$ that $\langle e, i\rangle=\left\langle e_{s}, i_{s}\right\rangle$, thus establishing (2.7). In (2.8) fix $s$ and $t>s$, and assume that 
$\left\langle e_{s}, i_{s}\right\rangle$ and $\left\langle e_{i}, i_{t}\right\rangle$ are defined, and that the antecedent holds. Now $v\left(s, e_{s}, i_{s}\right) \leqq v\left(t, e_{s}, i_{s}\right)$ by (2.3), and thus $\left\langle e_{t}, i_{t}\right\rangle\left\langle\left\langle e_{s}, i_{s}\right\rangle\right.$ by (2.2). If $n=v\left(s, e_{s}, i_{s}\right)$, then $n \in A^{s+1}-A^{s}$ implies $\sim V\left(t+1, e_{s}, i_{s}, n\right)$ because $t>s$. Hence, by the definition of $v, v\left(t+1, e_{s}, i_{s}\right) \neq n$. Thus by (2.3), $n=v\left(s, e_{s}, i_{s}\right)<v\left(t+1, e_{s}, i_{s}\right)$.

By (2.4) we know that $\Phi\left(A^{s+1}\right) \geqq \Phi\left(A^{s}\right)$ for all $s$. Hence, $\lim _{s} A^{s}$ must exist and will be denoted by $A$. That $A$ is infinite will follow by Lemma 2.3 .

Lemma 2.2. $L(A)$ is stably recursively enumerable.

Proof. By Lemma 1.1 and the above $L(A)$ is r.e. because the sequence $\left\{A^{s}\right\}$ satisfies (1.1) and (1.2). To prove that $L(A)$ is stably r.e. fix $n \in A$, and suppose that $n \in A^{s+1}-A^{s}$. Then $n=v\left(s, e_{s}, i_{s}\right)$ by (2.5). Now suppose for some $t>s$ that $n \in A^{t}-A^{t+1}$, and that $t^{\prime}$ is the least such $t$. Necessarily $n>v\left(t^{\prime}, e_{t^{\prime}}, i_{t^{\prime}}\right)$. Now by (2.8) and (2.3), $(u)_{>t^{\prime}}\left[n \neq v\left(u, e_{s}, i_{s}\right)\right]$. But then by $(2.7),(u)_{>t^{\prime}}(e)(i)[n \neq v(u, e, i)]$, and thus $(u)_{>t},\left[n \in A^{u}\right]$.

Lemma 2.3. For all $e$ and $i, \lim _{s} v(s, e, i)$ exists (and is denoted by $v(e, i))$, and $A=\left\{v(e, i) \mid\right.$ card $\left.\left.W_{e}\right\rangle\langle e, i+1\rangle\right\}$.

Proof. We prove both parts simultaneously by induction on $\langle e, i\rangle$. If $\langle e, i\rangle=0$, then $e=i=0$, and $v(s, 0,0)=v(0,0)$ for all $s$. Furthermore, clearly

$$
\left.v(0,0) \in A \Leftrightarrow(\exists s)\left[\operatorname{card} W_{0}^{s}\right\rangle\langle 0,1\rangle\right] .
$$

Fix $e$ and $i$, and assume by induction that the lemma holds for all $x$ and $y$ such that $\langle x, y\rangle\langle\langle e, i\rangle$. Now define,

$$
\begin{aligned}
s^{\prime}= & \mu s(t)_{\geqq s}(x)(y)[\langle x, y\rangle\langle\langle e, i\rangle \\
& \left.\Rightarrow[v(t, x, y)=v(x, y)] \&\left[v(x, y) \in A \Leftrightarrow v(x, y) \in A^{t}\right]\right] .
\end{aligned}
$$

Then $v\left(s^{\prime}, e, i\right)=v(e, i)$ because if $v(s+1, e, i)>v(s, e, i)$ for some $s \geqq s^{\prime}$, then by (2.6), $n \in A^{s+1}-A^{s}$ for some $n<v(s, e, i)$. But by (2.5), $n=v\left(s, e_{s}, i_{s}\right)$, and by (2.2), $\left\langle e_{s}, i_{s}\right\rangle\langle\langle e, i\rangle$ contradicting the definition of $s^{\prime}$.

Before proving the second half of the lemma note that for all $s$ and $n$,

$$
\left[n \in A \& n \in A^{s+1}-A^{s}\right] \Longrightarrow n=v\left(s, e_{s}, i_{s}\right)=v\left(e_{s}, i_{s}\right)
$$

because $n=v\left(s, e_{s}, i_{s}\right)$ by $(2.5)$, but if $v\left(s, e_{s}, i_{s}\right)<v\left(t, e_{s}, i_{s}\right)$ for some $t>s$, then $(u)_{\geq t}\left[n \in A^{u}\right]$ by the proof of Lemma 2.2.

Now suppose $v(e, i) \in A$, say $v(e, i) \in A^{s+1}-A^{s}$. Then $v(e, i)=$ 
$v(s, e, i)=v\left(s, e_{s}, i_{s}\right)$ by $(2.9)$. Hence, $\langle e, i\rangle=\left\langle e_{s}, i_{s}\right\rangle$, and card $\left.W_{e}^{s}\right\rangle\langle e, i+1\rangle$ by the eligibility of $\langle e, i\rangle$ at $s$.

Conversely let $t^{\prime}=(\mu t)_{>s^{\prime}}$ [card $\left.\left.W_{e}^{t}\right\rangle\langle e, i+1\rangle\right]$, where $s^{\prime}$ is defined as above. If $v\left(t^{\prime}, e, i\right) \notin A^{t^{\prime}}$ already, then $\langle e, i\rangle$ is eligible at $t^{\prime}$, and is the least eligible at $t^{\prime}$ by the definition of $s^{\prime}$. Hence, $v\left(t^{\prime}, e, i\right) \in A^{t^{\prime}+1}$, and $v\left(t^{\prime}, e, i\right)=v(e, i)$ by (2.6) since $t^{\prime} \geqq s^{\prime}$. Finally, $v(e, i) \in A$ because if $v(e, i) \in A^{t}-A^{t+1}$ for some $t>t^{\prime}$, then $v(t, x, y) \in A^{t+1}-A^{t}$ for some $\langle x, y\rangle\left\langle\langle e, i\rangle\right.$ contradicting the definition of $s^{\prime}$.

Before proceding to Lemma 2.4, we note that by (2.2),

$$
(e)(i)(x)(y)[v(e, i)<v(x, y) \Leftrightarrow\langle e, i\rangle\langle\langle x, y\rangle] .
$$

Now from (2.10) and the second part of Lemma 2.3,

$$
(x)(y)[a(\langle x, y\rangle) \geqq v(x, y)]
$$

where $a(n)$ is the principal function of $A$.

LEMмA 2.4. For all $e$, if $W_{e}$ is infinite, then

$$
(n)[\langle e, 0\rangle<n \Rightarrow a(n) \geqq h(e, n)] \text {. }
$$

Proof. If false, let $e, i$, and $n$ be such that $W_{e}$ is infinite, and $\langle e, i\rangle\langle n \leqq\langle e, i+1\rangle$, and $a(n)<h(e, n)$. Now $v(e, i) \in A$ by Lemma 2.3 since $W_{e}$ is infinite. Let $v(e, i) \in A^{s+1}-A^{s}$. Then by $(2.9), v(e, i)=$ $v(s, e, i)=v\left(s, e_{s}, i_{s}\right)$, and thus $\langle e, i\rangle=\left\langle e_{s}, i_{s}\right\rangle$. Let $n=\langle x, y\rangle$. Since $\langle e, i\rangle\langle\langle x, y\rangle \leqq\langle e, i+1\rangle$, we have by the second clause in the definition of $v$,

$$
v(s+1, x, y) \geqq h(s, e,\langle x, y\rangle) .
$$

Now by (2.11) and (2.3) respectively,

$$
\begin{aligned}
& a(\langle x, y\rangle) \geqq v(x, y) \geqq v(s+1, x, y), \text { and } \\
& h(s, e,\langle x, y\rangle) \geqq h(e,\langle x, y\rangle), \text { by }(2.1) .
\end{aligned}
$$

Arranging in order the inequalities of (2.13), (2.12) and (2.14) respectively, we conclude that $a(\langle x, y\rangle) \geqq h(e,\langle x, y\rangle)$, that is $a(n) \geqq h(e, n)$, contrary to hypothesis.

3. A cohesive set with recursively enumerable lower cut. An infinite set $C$ is cohesive if there is no r.e. set $W_{e}$ such that $W_{e} \cap C$ and $\bar{W}_{e} \cap C$ are both infinite. An r.e. set $M$ is maximal if $\bar{M}$ is cohesive. Although the construction of a maximal set requires a priority argument, it is easy to give a noneffective construction of a cohesive set (which is not co-r.e.). (The following in substance is the 
construction of Dekker and Myhill which appears in Rogers [6], p. 232.) Define a sequence of indices, $e_{0}, e_{1} \cdots$, as follows:

$$
\begin{aligned}
e_{0} & =\mu e\left[W_{e} \text { is infinite }\right] \\
e_{i+1} & =(\mu e)_{>e_{i}}\left[W_{e} \cap S_{i} \text { is infinite }\right], \text { where } S_{i}=\cap\left\{W_{e_{j}} \mid j \leqq i\right\} .
\end{aligned}
$$

Now define $C=\bigcup_{i}\left\{x_{i}\right\}$ where $x_{i}$ is some element of $S_{i}$, then $C$ is clearly cohesive since

$$
(e)\left[W_{e} \cap C \text { infinite } \Rightarrow C \subset{ }^{*} W_{e}\right] .
$$

(Recall that $A \subset{ }^{*} B$ denotes that $B-A$ is finite.)

This procedure is so noneffective, however, that it has rarely been used in an effective construction of some r.e. set. (For instance, the usual co-maximal cohesive sets $C$ given by the Yates construction (see Rogers [6]) do not satisfy the property that $C \subset{ }^{*} S_{i}$ for every $i$.) We will construct a cohesive set $A$ such that $L(A)$ is r.e., and such that for every $i, A \subset{ }^{*} S_{i}$. The latter property guarantees that $A$ is cohesive because if $A \cap W_{e}$ is infinite, then $e=e_{i}$ for some $i$, but then $A \subset{ }^{*} S_{i}$, and hence $A \subset{ }^{*} W_{e_{i}}$. (Throughout the proof we will refer to the indices $\left\{e_{i}\right\}$ and the sets $\left\{S_{i}\right\}$ defined above.)

THEOREm 3.1. ${ }^{1}$ There is an infinite set $A$ such that $L(A)$ is r.e., and $A \subset{ }^{*} S_{i}$ for every $i$ (and hence $A$ is cohesive).

(Again our proof will be an attempt to satisfy certain "requirements". Requirement $x$, denoted $R_{x}$, states that,

$$
A \subset * \cap\left\{W_{j} \mid j \in D_{x}\right\} \text {. }
$$

Naturally, it will be impossible to simultaneously satisfy all requirements, but we will prove (Lemma 3.8) that if " $D_{x}=\left\{e_{0}, e_{1}, \cdots, e_{i}\right\}$ for some $i$, then $R_{x}$ is satisfied, i.e., that

$$
A \subset{ }^{*} \cap\left\{W_{j} \mid j \in D_{x}\right\}=S_{i} .
$$

We say $R_{x}$ has higher priority than $R_{y}$ just if $\Phi\left(D_{x}\right)>\Phi\left(D_{y}\right)$. To aid intuition one may imagine that a "marker" $\Lambda_{x}$ corresponds to $R_{x}$ for every $x$, and that $v(s, x)$ denotes the integer occupied by $\Lambda_{x}$ at stage $s$. Ideally, we would like to reflect the priority of requirements as in (2.2) by defining $v(s, x)$ so that for all $s, v(s, x)<v(s, y) \Leftrightarrow \Phi\left(D_{x}\right)>\Phi\left(D_{y}\right)$, because the leftmost markers (i.e., markers occupying smaller integers) will exercise greatest control over elements eventually admitted to $A$. Naturally, this is impossible since markers would have an infinite number of predecessors. We must therefore begin more modestly with

${ }^{1}$ This question was suggested to us by T.G. McLaughlin. 
a recursive well ordering of type $\omega, W(x, y)$, and then allow markers to change their relative positions so as to more closely approximate the priority ordering when desirable in order to attempt to satisfy a certain requirement.)

Proof. From now on we adopt the convention that $\max D_{x}$ denotes $\max \left[n \mid n \in D_{x}\right]$, and $\max \varnothing=0$. Define the recursive predicate,

$$
W(x, y) \equiv\left\{\max D_{x}<\max D_{y}\right\} \vee\left[\max D_{x}=\max D_{x} \& \Phi\left(D_{x}\right)>\Phi\left(D_{y}\right)\right] .
$$

We define a canonically r.e. sequence of finite sets, $\left\{A^{s}\right\}$, and a recursive function $v(s, x)$ as follows. Set $A^{0}=\varnothing, v(0,0)=0$, and for $x>1$. define

$$
v(0, x)=\mu n(y)[W(y, x) \Rightarrow v(0, y)<n] .
$$

Stage $s \geqq 0$. Define the function $f$,

$$
f(s, x)=\max \left\{\cup D_{y} \mid \text { all } y \text { such that } v(s, y) \leqq v(s, x)\right\} .
$$

(That $f$ is recursive will follow because $v$ will be recursive and because $\lambda y v(s, y)$ will be a one-one function.) We define $x$ to be eligible at stage $s$, denoted $E(s, x)$, as follows:

$$
E(s, x) \equiv \operatorname{card}\left\{n \mid n>v(s, x) \& n \in \cap\left\{W_{i}^{s} \mid i \in D_{x}\right\}\right\}>2^{f(s, x)+2} .
$$

Case 1. There is no eligible $x$ at stage $s$. Then set $A^{s+1}=A^{s}$, and $v(s+1, x)=v(s, x)$ for all $x$, and go to stage $s+1$. (Note that $(\exists x) E(s, x)$ is decidable given $\lambda x v(s, x)$ since one need only examine those $x$ such that $v(s, x)<s$, because $(j)(z)_{\geqq s}\left[z \notin W_{j}^{s}\right]$ by the Gödel numbering.)

Case 2. Otherwise. Let $x_{s}$ be the unique eligible $x$ which satisfies the predicate

$$
L(s, x) \equiv E(s, x) \& \sim(\exists y)[E(s, y) \& v(s, y)<v(s, x)] .
$$

(That is, $x_{s}$ is the unique eligible $x$ whose marker $\Lambda_{x}$ is leftmost among all the markers $\Lambda_{y}$ such that $y$ is eligible at $s$.)

Now let $m_{s}=f\left(s, x_{s}\right)+1$, and define the sets,

$$
\begin{aligned}
& X_{1}^{s}=\left\{x \mid v(s, x)<v\left(s, x_{s}\right)\right\} \\
& X_{2}^{s}=\left\{x \mid v(s, x) \geqq v\left(s, x_{s}\right) \& D_{x} \subseteq I\left[0, m_{s}\right]\right\} \\
& X_{3}^{s}=\left\{x \mid v(s, x) \geqq v\left(s, x_{s}\right) \& D_{x} \nsubseteq I\left[0, m_{s}\right]\right\} .
\end{aligned}
$$

Note that card $X_{2}^{s} \leqq 2^{f\left(s, x_{s}\right)+2}$. (Viewing the following definition of $v(s+1, x)$ as a description of how the markers move, notice that only 
the markers $\Lambda_{x}$ for $x \in X_{2}^{s}$ are allowed to change their relative order, and they move only so as to more closely approximate our priority ranking. Furthermore, since the elements $v(s+1, x)$ are potential elements of $A^{t}$ for some $t>s+1$, the first conjunct of the case $x \in X_{2}^{s}$ attempts to partially satisfy requirement $R_{x_{s}}$ ). Define,

$$
\begin{aligned}
& A^{s+1}=\left[A^{s} \cap I\left[0, v\left(s, x_{s}\right)\right] \cup\left\{v\left(s, x_{s}\right)\right\}\right. \text {, and } \\
& v(s+1, x)= \begin{cases}v(s, x) & \text { if } x \in X_{1}^{s} \\
\mu n\left[n \in \cap\left\{W_{i}^{s} \mid i \in D_{x_{s}}\right\} \& n>v(s, x)\right. & \text { if } x \in X_{2}^{s} \\
\left.\&(y)\left[\left[y \in X_{2}^{s} \& \Phi\left(D_{y}\right)>\Phi\left(D_{x}\right)\right] \Rightarrow v(s+1, y)<n\right]\right] & \\
\mu n(y)\left[\left[y \in X_{2}^{s} \vee\left[y \in X_{3}^{s} \& v(s, y)<v(s, x)\right]\right]\right. & \text { if } x \in X_{3}^{s} \\
\Rightarrow v(s+1, y)<n] . & \end{cases}
\end{aligned}
$$

(It is clear by recursion on $s$ that the function $v(s, x)$ is recursive since $\lambda x v(s+1, x)$ is uniformly recursive in $\lambda x f(s, x), E(s, x)$, and $X_{i}^{s}, 1 \leqq i \leqq 3$, which in turn are uniformly recursive in $\lambda x v(s, x)$.)

By the definition of $v(s+1, x)$ we have for all $s, x, y$ and $z$,

$$
v(s, x) \neq v(s, y) \Longrightarrow x \neq y
$$

(3.2) $x \in X_{1}^{s} \& y \in X_{2}^{s} \& z \in X_{3}^{s} \Rightarrow v(s+1, x)<v(s+1, y)<v(s+1, z)$

(3.3) $v(s, x)<v(s, y) \& v(s+1, x)>v(s+1, y) \Rightarrow x \in X_{2}^{s} \& y \in X_{2}^{s}$

$$
x \in X_{2}^{s} \Rightarrow v(s+1, x) \in \cap\left\{W_{i}^{s} \mid i \in D_{x_{s}}\right\} .
$$

To see (3.2), suppose $x \in X_{1}^{s}$ and $y \in X_{2}^{s}$, then $v(s+1, y)>v(s, y) \geqq$ $v\left(s, x_{s}\right)>v(s, x)=v(s+1, x)$. The rest of (3.2) is clear, while (3.3) follows from (3.2) and the fact that if $x, y \in X_{1}^{s}$ or $x, y \in X_{3}^{s}$ then $v(s, x)<v(s, y)$ if and only if $v(s+1, x)<v(s+1, y)$. Finally, (3.4) follows by the definition of $v(s+1, x)$.

By the definitions of $v$ and $f$, we have for all $s$ that if $(\exists x) L(s, x)$, i.e., if $x_{s}$ is defined, then

$$
f\left(s, x_{s}\right)<f\left(s+1, x_{s}\right)
$$

because if $D_{y}=D_{x_{s}} \cup\left\{m_{s}\right\}$ then $y \in X_{2}^{s}$, and so by the second clause in the definition of $v, v(s+1, y)<v\left(s+1, x_{s}\right)$ because $\Phi\left(D_{y}\right)>\Phi\left(D_{x_{s}}\right)$. But then $f\left(s+1, x_{s}\right) \geqq m_{s}=1+f\left(s, x_{s}\right)$.

Furthermore, it is clear that for all $x, n$ and $s$,

$$
\begin{gathered}
n \in A^{s+1}-A^{s} \Leftrightarrow n=v\left(s, x_{s}\right) \\
v(s, x) \neq v(s+1, x) \Rightarrow(\exists m)\left[m<v(s, x) \& m \in A^{s+1}-A^{s}\right] \\
n \in A^{s}-A^{s+1} \Rightarrow(\exists m)\left[m<n \& m \in A^{s+1}-A^{s}\right] .
\end{gathered}
$$

Using (3.6) and the fact that $v\left(s+1, x_{s}\right)>v\left(s, x_{s}\right)$ (because $\left.x_{s} \in X_{2}^{s}\right)$, 
it is easily seen by induction on $s$ that

$$
(s)(x)\left[v(s, x) \notin A^{s}\right] \text {. }
$$

Now by (3.9) and the definition of $A^{s+1}$, we have $\Phi\left(A^{s+1}\right) \geqq \Phi\left(A^{s}\right)$ for all $s$. Thus $\lim _{s} A^{s}$ must exist, and will be denoted by $A$. Since the canonically r.e. sequence, $\left\{A^{s}\right\}$, of finite sets satisfies (1.1) and (1.2), we have proved.

Lemma 3.2. $L(A)$ is r.e.

(Of course, unlike the sequence in Theorem 2.1, we know that $\left\{A^{\star}\right\}$ cannot satisfy (1.3) because no cohesive set may contain an infinite retraceable subset.)

For future reference we will define the nonrecursive function $s$,

$$
s(n)=\mu t(m)_{<n+1}\left[m \in A \Leftrightarrow m \in A^{t+1}\right] .
$$

By (3.8) and the definition of $s(n)$ we have,

$$
(t)_{>s(n)}(m)_{<n+1}\left[m \in A \Leftrightarrow m \in A^{t}\right] .
$$

Finally, by the minimality of $s(n)$ we see that if $n \in A$, then $n \in A^{s(n)+1}-A^{s(n)}$, so that by (3.6),

$$
(n)\left[n \in A \Leftrightarrow n=v\left(s(n), x_{s(n)}\right)\right] .
$$

Lemma 3.3. $A$ is an infinite set.

Proof. If $A$ is finite, let $m \equiv \max \{n \mid n \in A\}$. Then by (3.11) and (3.7),

$$
(x)(t)_{>s(m)}\left[A=A^{t} \& v(t, x)=v(s(m)+1, x)\right] .
$$

But since there are an infinite number of $x$ such that $\cap\left\{W_{i} \mid i \in D_{x}\right\}$ is infinite, there must exist some $t>s(m)$ and some $x$ such that $x$ is eligible at stage $t$. But then $v\left(t, x_{t}\right) \in A^{t+1}-A^{t}$, contradicting $A^{t+1}=$ $A=A^{t}$ for $t>s(m)$.

Lemma 3.4. For all $x \neq 0$, if $\cap\left\{W_{i} \mid i \in D_{x}\right\}$ is finite, then $\left\{s \mid(\exists y)\left[D_{y} \supseteqq D_{x} \& L(s, y)\right]\right\}$ is finite also.

Proof. Fix $x \neq 0$. Let $m=\max \left\{n \mid n \in\left\{W_{i} \mid i \in D_{x}\right\}\right\}$. (Recall that $\max \varnothing=0$.) Then

$$
(y)(t)_{>s(m)}\left[D_{y} \supseteqq D_{x} \Rightarrow \sim L(t, y)\right],
$$

because if $v(t, y) \leqq m$ and $L(t, y)$ then $v(t, y) \in A^{t+1}-A^{t}$ contradicting 
(3.11). But if $v(t, y)>m$, then $\sim L(t, y)$ because $\sim E(t, y)$ since $\cap\left\{W_{i}^{t} \mid i \in D_{y}\right\} \subseteq I[0, m]$.

Now define a (nonrecursive) function $d$ as follows:

$$
D_{d(i)}=\left\{e_{0}, e_{1}, \cdots, e_{i}\right\}
$$

where $e_{1}, e_{1}, \ldots$ is the sequence of indices defined in the beginning of §3. (Note that $S_{i}=\cap\left\{W_{i} \mid j \in D_{d(i)}\right\}$.)

Lemma 3.5. $(n)[n \in A \Rightarrow n \leqq v(s(n), d(i))]$.

Proof. Suppose that $n>v(s(n), d(i))$. Now by (3.7) and (3.11), $v(t, d(i))=v(s(n), d(i))$ for all $t>s(n)$. Now since $\cap\left\{W_{j} \mid j \in D_{d(i)}\right\}$ is infinite, there must be some $t>s(n)$ such that $d(i)$ is eligible at stage $t$. But then $L(t, y)$ holds for some $y$ such that $v(t, y) \leqq v(t, d(i))$, and hence $m \in A^{t+1}-A^{t}$ for some $m<v(s(n), d(i))$, contradicting (3.11).

LEMMA 3.6. For all $s, x$, and $y$,

$$
v(s, x)<v(s, y) \Rightarrow\left[\max D_{x}<\max D_{y} \vee \Phi\left(D_{x}\right)>\Phi\left(D_{y}\right)\right] .
$$

Proof. This is clearly true for $s=0$ by definition of $\lambda x v(0, x)$. Assume true for some fixed $s$, and suppose $v(s+1, x)<v(s+1, y)$. Now if $v(s, x)<v(s, y)$ then the conclusion follows by inductive hypothesis. But by (3.3) if $v(s, x)>v(s, y)$, then $x, y \in X_{2}^{s}$, and thus $v(s+1, x)<v(s+1, y)$ only if $\Phi\left(D_{x}\right)>\Phi\left(D_{y}\right)$.

Lemma 3.7. For every $i$, there exists $t_{i}$ such that

$$
(s)_{>t_{i}}(x)\left[L(s, x) \& v(s, x) \leqq v(s, d(i)) \Rightarrow D_{x} \supseteqq D_{d(i)}\right] .
$$

Proof. The proof is by induction on $i$.

Case $i=0$. Define

$$
t_{0}=\max \left\{t \mid(\exists j)(\exists y)\left[j<e_{0} \&\{j\} \subseteq D_{y} \& L(t, y)\right]\right\},
$$

which is at most a finite set by Lemma 3.4. Now by Lemma 3.6, for all $s$ and $x$,

$$
\begin{aligned}
& v(s, x)<v(s, d(0)) \Rightarrow\left[\max D_{x}<d(0) \vee \Phi\left(D_{x}\right)>\Phi\left(D_{d(0)}\right)\right] \\
& \therefore(s)_{>t_{0}}(x)\left[L(s, x) \& v(s, x) \leqq v(s, d(0)) \Rightarrow D_{x} \supseteqq D_{d(0)}\right] .
\end{aligned}
$$

Case $i+1$. By induction, assume that for all $j \leqq i, t_{j}$ is defined so that the above statement holds. Define

$$
w=\max \left\{s \mid(\exists j)(\exists y)\left[e_{i}<j<e_{i+1} \& D_{y} \supseteqq D_{d(i)} \cup\{j\} \& L(t, y)\right]\right\}
$$


which is at most a finite set by Lemma 3.4, and the definition of $e_{i+1}$. Define $r=\max \left\{t_{i}, w\right\}$. Thus,

$$
(s)_{>r}(x)\left[[L(s, x) \& v(s, x)<v(s, d(i))] \Longrightarrow D_{x} \supseteqq D_{d(i+1)}\right]
$$

because by inductive hypothesis and (3.1), $D_{x} \nsupseteq D_{d(i)}$, and by (3.13), $D_{x} \nsupseteq D_{d(i)} \cup\{j\}$ for any $j<e_{i+1}$. (That $D_{x} \nsupseteq D_{d(i)} \cup\{j\}$ for $j<e_{i}$ and $j \notin D_{d(i)}$ follows of course by inductive hypothesis.)

Subcase 1. $(\exists s)_{>r}[v(s, d(i+1))<v(s, d(i))]$. If $u$ is the least such $s$, then a second induction on $s$ for $s \geqq u$ proves simultaneously that,

$$
(s)_{\geqq u}[v(s+1, d(i+1))<v(s+1, d(i))] \text {, and }
$$

$$
(s)_{\geqq u}(x)\left[L(s, x) \& v(s, x) \leqq v(s, d(i+1)) \Rightarrow D_{x} \supseteq D_{d(i+1)}\right] \text {. }
$$

By the definition of $u$, we have $v(u, d(i+1))<v(u, d(i))$. Choose $t \geqq u$, and assume (3.15) and (3.16) for all $s$ such that $u \leqq s<t$. We may assume that,

$$
(\exists x)[L(t, x) \& v(t, x) \leqq v(t, d(i+1))]
$$

because otherwise $v(t+1, y)=v(t, y)$ for all $y$, such that $v(t, y) \leqq$ $v[t, d(i))$, and (3.15) and (3.16) hold trivially for $s=t$. Now by (3.14), $D_{x} \supseteq D_{d(i+1)}$ thus establishing (3.16) for $s=t$.

To prove (3.15) for $s=t$, note that $f\left(t, x_{t}\right) \geqq e_{i+1}$ by the definition of $f$ since $D_{x_{t}} \supseteqq D_{d(i+1)}$. But then $d(i), d(i+1) \in X_{2}^{t}$ because $D_{d(i+1)} \cong$ $I\left[0, f\left(t, x_{t}\right)+1\right], \quad$ and $\quad v\left(t, x_{t}\right) \leqq v(t, d(i+1))<v(t, d(i))$. Hence, $v(t+1, d(i+1))<v(t+1, d(i))$ by the second clause in the definition of $v$ because $\Phi\left(D_{d(i+1)}\right)>\Phi\left(D_{d(i)}\right)$.

Subcase 2. $(s)_{>r}[v(s, d(i))<v(s, d(i+1))]$. This assumption will lead to a contradiction. Define

$$
u(1)=(\mu s)_{>r}(\exists y)[L(s, y) \& v(s, y) \leqq v(s, d(i))] .
$$

(Such an $s$ exists by Lemma 3.5 and (3.12) since $A$ is infinite and $A^{r}$ is finite.) Now by (3.1) and (3.14), $D_{x_{u(1)}} \supseteq D_{d(i+1)}$ or $x_{u(1)}=d(i)$. But if the former then $d(i), d(i+1) \in X_{2}^{u(1)}$, because

$$
v\left(u(1), x_{u(1)}\right) \leqq v(u(1), d(i))<v(u(1), d(i+1)) .
$$

But then since $\Phi\left(D_{d(i+1)}\right)>\Phi\left(D_{d(i)}\right)$ we have by the definition of $v$ that

$$
v(u(1)+1, d(i+1))<v(u(1)+1, d(i))
$$

contrary to the hypothesis.

We conclude that $x_{u(1)}=d(i)$. But then by (3.5),

$$
f(u(1)+1, d(i))>f(u(1), d(i)) \geqq e_{i} .
$$

Now define, 


$$
u(2)=(\mu s)_{>u(1)}(\exists y)[L(s, y) \& v(s, y) \leqq v(s, d(i))] .
$$

By the same argument as above, $x_{u(2)}=d(i)$, and

$$
f(u(2)+1, d(i))>f(u(2), d(i))>f(u(1), d(i)) \geqq e_{i} .
$$

Continuing in this manner, after at most $k=e_{i+1}-e_{i}$ steps, we must have $f(u(k), d(i)) \geqq e_{i+1}-1$. But then $D_{d(i+1)} \leqq I\left[0, m_{u(k)}\right]$ so that $d(i), d(i+1) \in X_{2}^{u(k)}$. Thus by the definition of $v$,

$$
v(u(k)+1, d(i+1))<v(u(k)+1, d(i)),
$$

contradicting the assumption of subcase 2 .

Thus if we define

$$
t_{i+1}=(\mu s)_{>r}[v(s, d(i+1))<v(s, d(i))]
$$

then Lemma 3.7 follows.

Lemma 3.8. For every $i, A \subset{ }^{*} S_{i}$.

Proof. Fix $i$, and let $t_{i}$ be defined as in Lemma 3.7. Let $n=$ $\mu m\left[m \in A-A^{t_{i}}\right]$. By (3.12), $n=v\left(s(n), x_{s(n)}\right)$, and $s(n)>t_{i}$ since $n \notin A^{t_{i}}$. By Lemma 3.5, $n<v(s(n), d(i))$. Now $v\left(s(n), x_{s(n)}\right)<v(s(n), d(i))$ implies by Lemma 3.7 that $D_{x_{s(n)}} \supseteqq D_{d(i)}$. Hence, $d(i) \in X_{2}^{s(n)}$. But then by (3.2) and the definition of $v$ we have for all $y$,

$$
n<v(s(n)+1, y) \leqq v(s(n)+1, d(i)) \Rightarrow y \in X_{2}^{s(n)} .
$$

Thus, by the second clause in the definition of $v$, we have for all $y$ and for $t=s(n)+1$,

$$
n<v(t, y) \leqq v(t, d(i)) \Rightarrow v(t, y) \in \cap\left\{W_{j} \mid j \in D_{x_{s(n)}}\right\} .
$$

But since $D_{x_{s}} \supseteqq D_{d(i)}$, we have for all $y$, and for $t=s(n)+1$,

$$
n<v(t, y) \leqq v(t, d(i)) \Rightarrow v(t, y) \in S_{i} .
$$

Now we will prove by induction on $t$ that (3.17) holds for all $t \geqq s(n)+1$. This will prove that

$$
(m)_{>n}\left[m \in A \Rightarrow m \in S_{i}\right]
$$

because if $m \in A$ then by (3.12) $m=v\left(s(m), x_{s(m)}\right)$. But $m>n$ implies $s(m) \geqq s(n)+1$. Now by Lemma 3.5 ,

$$
v\left(s(m), x_{s(m)}\right)=m<v(s(m), d(i)) .
$$

Hence, $v\left(s(m), x_{s(m)}\right) \in S_{i}$ by (3.17).

It remains to prove (3.17) by induction on $t \geqq s(n)+1$. Since 
(3.17) clearly holds for $t=s(n)+1$, choose $u \geqq s(n)+1$ and assume by induction that (3.17) holds for all $t \leqq u$. Now (3.17) follows trivially for $t=u+1$ by inductive hypothesis and the definition of $v$ unless,

$$
(\exists y)[L(u, y) \& v(u, y) \leqq v(u, d(i))] .
$$

In this case by Lemma 3.7, $D_{x_{u}} \supseteqq D_{d(i)}$ since $u>s(n)>t_{i}$. But $D_{x_{u}} \supseteq D_{d(i)}$ implies $d(i) \in X_{2}^{u}$. Thus by (3.2) for all $y$,

$$
v(u+1, y)<v(u+1, d(i)) \Rightarrow\left[y \in X_{1}^{u} \vee y \in X_{2}^{u}\right] \text {. }
$$

Now if $y \in X_{1}^{u}$, then $v(u+1, y)=v(u, y)$ and so if $n<v(u, y)$, then $v(u+1, y) \in S_{i}$ by inductive hypothesis. But $y \in X_{2}^{u}$ implies $v(u+1, y) \in \cap\left\{W_{j} \mid j \in D_{x_{u}}\right\}$ by (3.4). Hence, since $D_{x_{u}} \supseteqq D_{d(i)}, v(u+$ $1, y) \in S_{i}$.

\section{BIBLIOGRAPHY}

1. J. C. E. Dekker and J. Myhill, Retraceable sets, Canad. J. Math. 10 (1958), 357-373.

2. D. A. Martin, A theorem on hyperhypersimple sets, J. of Symbolic Logic 28 (1963), 273-278.

3. - Classes of recursively enumerable sets and degrees of unsolvability, Zeitschr.

F. Math Logik und Grundl. Math. 12 (1966), 295-310.

4. H. G. Rice, Recursive real numbers, Proc. Amer. Math. Soc. 5 (1954), 784-791.

5. Recursive and recursively enumerable orders, Trans. Amer. Math. Soc. 83 (1956), 277-300.

6. H. Rogers, Jr., Theory of recursive functions and effective computability, McGrawHill, New York, 1967.

7. G. E. Sacks, Degrees of unsolvability, Ann. of Math. Study 55, Princeton, 1963.

8. R. I. Soare, Recursion theory and Dedekind cuts, (to appear in Trans. Amer. Math. Soc.)

9. C. E. M. Yates, Recursively enumerable sets and retracing functions, Zeitschr. f. math. Logik und Grundl. Math. 8 (1962), 331-345.

Received June 27, 1967, and in revised form February 18, 1969. These results are part of the author's doctoral dissertation written at Cornell University under the direction of Professor Anil Nerode, and with helpful criticism by Professor T. G. McLaughlin. The results were presented to the American Mathematical Society in April, 1967.

University of Illinois at Chicago Circle 



\section{PACIFIC JOURNAL OF MATHEMATICS}

\section{EDITORS}

H. ROYDEN

Stanford University

Stanford, California

Richard Pierce

University of Washington

Seattle, Washington 98105
J. DUGUNDJI

Department of Mathematics

University of Southern California

Los Angeles, California 90007

BASIL GORDON

University of California

Los Angeles, California 90024

\section{ASSOCIATE EDITORS}
E. F. BECKENBACH
B. H. NEUMANN
F. WOLF
K. YosHIDA

\section{SUPPORTING INSTITUTIONS}

UNIVERSITY OF BRITISH COLUMBIA

CALIFORNIA INSTITUTE OF TECHNOLOGY

UNIVERSITY OF CALIFORNIA

MONTANA STATE UNIVERSITY

UNIVERSITY OF NEVADA

NEW MEXICO STATE UNIVERSITY

OREGON STATE UNIVERSITY

UNIVERSITY OF OREGON

OSAKA UNIVERSITY

UNIVERSITY OF SOUTHERN CALIFORNIA
STANFORD UNIVERSITY

UNIVERSITY OF TOKYO

UNIVERSITY OF UTAH

WASHINGTON STATE UNIVERSITY

UNIVERSITY OF WASHINGTON

*

AMERICAN MATHEMATICAL SOCIETY CHEVRON RESEARCH CORPORATION TRW SYSTEMS

NAVAL WEAPONS CENTER 


\section{Pacific Journal of Mathematics}

\section{Vol. 31, No. $1 \quad$ November, 1969}

James Burton Ax, Injective endomorphisms of varieties and schemes........

Richard Hindman Bouldin, A generalization of the Weinstein-Aronszajn

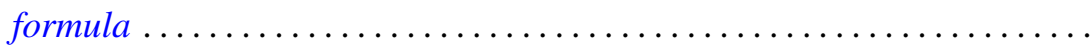

John Martin Chadam, The asymptotic behavior of the Klein-Gordon equation with external potential. II ...............................

Rina Hadass, On the zeros of the solutions of the differential equation

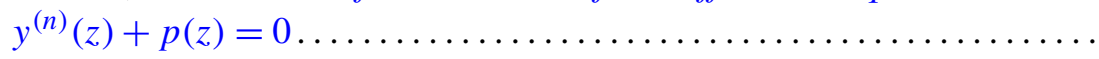

John Sollion Hsia, Integral equivalence of vectors over local modular lattices. II .............................................

Robert Hughes, Boundary behavior of random valued heat polynomial

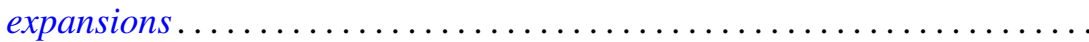

Surender Kumar Jain, Saad H. Mohamed and Surjeet Singh, Rings in which every right ideal is quasi-injective .........................

T. Kawata, On the inversion formula for the characteristic function .........

Erwin Kleinfeld, On right alternative rings without proper right ideals......

Robert Leroy Kruse and David Thomas Price, On the subring structure of

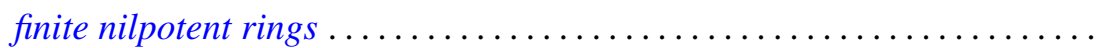

Marvin David Marcus and Stephen J. Pierce, Symmetric positive definite multilinear functionals with a given automorphism. .

William Schumacher Massey, Pontryagin squares in the Thom space of a bundle...

William Schumacher Massey, Proof of a conjecture of Whitney ...

John William Neuberger, Existence of a spectrum for nonlinear transformations

Stephen E. Newman, Measure algebras on idempotent semigroups ...

$\mathrm{K}$. Chandrasekhara Rao, Matrix transformations of some sequence spaces

Robert Bruce Schneider, Some theorems in Fourier analysis on symmetric

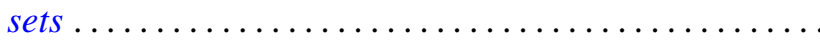

Ulrich F. K. Schoenwaelder, Centralizers of abelian, normal subgroups of hypercyclic groups...

Jerrold Norman Siegel, $G$-spaces, $H$-spaces and $W$-spaces

Robert Irving Soare, Cohesive sets and recursively enumerable Dedekind cuts...

Kwok-Wai Tam, Isometries of certain function spaces .... . .

Awadhesh Kumar Tiwary, Injective hulls of semi-simple modules over regular rings ....

Eldon Jon Vought, Concerning continua not separated by any nonaposyndetic subcontinuum .................... 